\title{
Markov Random Fields Based Probabilistic Relaying for Multihop Networks
}

\author{
Aruna Jayasuriya \\ CQUniversity \\ School of Engineering and Built Environment \\ Gladstone, Australia \\ Email:a.jayasuriya@cqu.edu.au
}

\author{
Sylvie Perreau \\ Institute for Telecommunications Research \\ University of South Australia \\ Mawson Lakes, Australia
}

\author{
Marc Sigelle \\ Institut TELECOM ParisTech \\ 46 Rue Barrault, Paris, France
}

\begin{abstract}
In this paper, we demonstrate how a Markov Random Field (MRF) based framework can be used for multihop networks analysis and design. In fields such as image processing it has been shown that MRFs is a powerful tool to analyse distributed systems with strong spacial interactions, which is also a defining characteristic of multihop networks. In this work we focus on using MRFs to model traffic intensity of sensor networks using shortest path routing. Later we propose a probabilistic relaying mechanism to recreate a traffic pattern similar to that observed in a network using shortest path routing. The objective is to emulate the shortest path performance without complex routing protocols and associated overheads. Using a simulation study we then show that the proposed mechanism achieves $95 \%$ of the throughput of shortest path, without using a routing protocol.
\end{abstract}

\section{INTRODUCTION}

Recent advances in mobile communication technologies, portable power sources, and high speed computing have resulted in an increasing interest in implementing and deploying ad hoc and sensor networks. Despite the significant research interest in the past decade, the current deployments of ad hoc and sensor networks are still largely at the experimental stage, mainly due to the lack of a solid theoretical understanding of such networks. So far, the majority of multihop network research activities have concentrated on the issues of designing new protocols such as Medium Access Control (MAC) [1], [2], routing [3], [4], transport [5], [6] or improving the performance of these networks by certain mechanisms or parameter optimisation [7], [8]. Evaluation of these protocols has mainly relied on simulation results [5], [9] and, to a lesser extent, analytical methods relying on simplified, often unrealistic, approximations [10], [11]. In most cases, these simulation results and simplified analytical studies only serve the purpose of comparing a new approach to an existing one. Furthermore, these studies have shown that the current protocols have severe scalability problems, where performance rapidly degrade with increasing number of nodes [6], [10].

It appears that most studies of multihop networks at various layers of the protocol stack face the fundamental issue of understanding complex interactions between nodes [12], [13]. In image processing where, similar to multihop networks, the spatial interactions in a 2 dimensional space is a crucial issue, Markov Fields have been successfully used to make use of local statistical interactions to simplify and solve many large scale problems [14], [15]. Hence, the use of a 2 dimensional Markovian structure seems a natural way to address the scalability issue of sensor and ad hoc networks.

In multihop networks, there are strong dependencies between neighbouring nodes, while there are often no direct interactions between non-neighbouring nodes. Hence, the lack of statistical tools to analyse large, distributed systems which exhibit strong spatial dependencies in a 2-dimensional domain is a major obstacle for developing a clear theoretical framework to understand, design and implement ad hoc and sensor networks. To overcome this problem, in this paper we use the theory of Markov Random Fields (MRFs) [16] to analyse the characteristics and performance of large sensor and ad hoc networks. Recently Markov Fields have been used to solve problems related multihop networking. In [17], authors develop a framework based on Hidden MRFs for distributed signal processing in sensor-network environments. This framework is used to extract underlying random fields that has a simple structure with Markovian dependence from data collected at different sensors. Doyle et. al. in [18] discuss how MRFs can be used to solve distributed decision making problems in ad hoc networks. More recently, in [19], authors use the idea of MRFs for the purpose of distributed configuration management in wireless networks. Although these related works do use MRF based framework, their focus is on the analysis of data retrieved from multihop networks for either detection or decision making purposes, as opposed our aim of using a MRF based framework to analyse and design protocols suited to multihop networks.

The objective of this paper is to capture the characteristics of traffic intensity at each node of a multihop network using shortest path routing. Then we aim to emulate that traffic pattern in an identical network using probabilistic retransmissions at each node instead of routing. The rest of the paper is organised as follows. Section II provide a brief summary on MRF theory, focusing on the aspects that are important for this study. This is followed by detailed description of the MRF based model used in this study to characterise the traffic intensity pattern in a multihop network. Section IV describes the relay mechanism proposed in the study to emulate the shortest path behaviour. The next section presents the simulation results, which shows the effectiveness of the proposed mechanism. This is followed by concluding remarks and suggestions for future work. 


\section{THEORY OF MARKOV RANDOM FIELDS}

Let us denote by $\mathbf{S}$ a set of sites with a neighbourhood system defined on it. For the sake of simplicity in this work, we model a 2-dimensional MRF as a rectangular grid where the neighbours of site $i$ are the 4 sites that are closest to it (4-neighbours), as shown in Figure 1. Let us denote by c, a set of sites in $\mathbf{S}$. This set $\mathbf{c}$ is called a clique if it contains several sites that are all neighbours to each other as indicated in figure 1. Let us define, $\mathbf{u}$, the collection of random variables defined on $\mathbf{S}$.

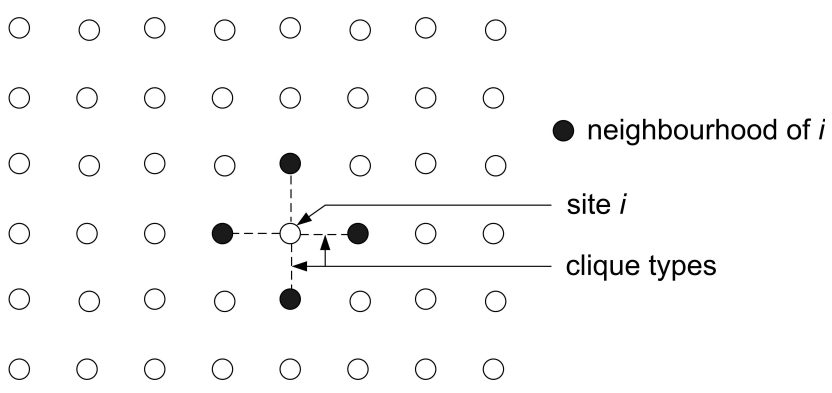

Fig. 1. Neighbourhood system

The set of variables $\mathbf{u}$ is called an MRF if all its realisations have nonzero probabilities and if its conditional distribution satisfies the following Markov property:

$$
p\left(\mathbf{u}_{i} \mid \mathbf{U}_{S-i}\right)=p\left(\mathbf{u}_{i} \mid \mathbf{U}_{j}, j \in \mathbf{N}_{i}\right)
$$

where $\mathbf{U}_{S-i}$ denotes the field restricted on $\mathbf{S}-i$ and $\mathbf{N}_{i}$ denotes the set of neighbours of $i$.

It is known that the joint probability distribution of $\mathbf{u}$ is a Gibbs distribution given by [16]:

$$
p(\mathbf{u})=Z^{-1} \exp [-\beta U(\mathbf{u})]
$$

where $U(\mathbf{u})$ is the energy function

$$
U(\mathbf{u})=\sum_{\mathbf{c}} \mathbf{V}_{\mathbf{c}}(\mathbf{u})
$$

and $\beta V_{\mathbf{c}}(\mathbf{u})$ are the "interaction functions" depending only on the restriction of $\mathbf{u}$ to the clique with the parameter $\beta$ adjusting the strength of the interaction.

A key feature of an MRF is that, even though it is not possible to evaluate the joint probability $p(\mathbf{u})$ in equation (2) because of the computational costs, it is possible to calculate the conditional probabilities $p\left(\mathbf{u}_{i} \mid \mathbf{U}_{j}, j \in \mathbf{N}_{i}\right)$ using:

$$
p\left(\mathbf{u}_{i} \mid \mathbf{U}_{j}, j \in \mathbf{N}_{i}\right)=\frac{\exp \left[-\beta V_{\mathbf{c}}\left(\mathbf{u}_{i}\right)\right]}{\sum_{j} \exp \left[-\beta V_{\mathbf{c}}\left(\mathbf{u}_{j}\right)\right]}
$$

\section{Modelling TrafFic InTEnsity}

In this paper we aim to find the traffic intensity of a node in a sensor network using a framework based on MRFs. We define traffic intensity of a node as the total traffic transmitted by a node, i.e, its own traffic plus the traffic it has to relay. The objective is to derive a relationship between the traffic intensity at each node, knowing the traffic intensity of each of the neighbouring nodes when a particular routing protocol is used. Once this relationship is understood, our next objective is to recreate the traffic intensity pattern in the network using a simple probabilistic packet relaying algorithm at each node, without using a routing algorithm to route packets between nodes. This will significantly reduce the routing overhead of the network without compromising the performance.

\section{A. Model Description}

Let us assume the the amount of traffic transmitted by node $i$ in the network, $u_{i}$ takes integer values such that:

$$
0 \leq u_{i} \leq M \forall i
$$

Let us define an energy function at node $i, T_{i}$ as:

$$
T_{i}=\beta_{1 i}+\beta_{2 i} \sum_{j \in \mathbf{N}_{i}} u_{j}
$$

where $\beta_{1 i}$ and $\beta_{2 i}$ are parameters that depends on a node's own traffic and neighbours' traffic intensities respectively and $\mathbf{N}_{i}$ is the neighbourhood of $i$. Let us assume a binomial distribution for each traffic intensity random variable $u_{i}$, conditional on the traffic intensity of neighbouring nodes $u_{j}, j \in \mathbf{N}_{i}$. Then the conditional distribution of traffic intensities at nodes can be written as:

$$
P\left(u_{i}=n \mid u_{j}, j \in N_{i}\right)=C_{M}^{n}\left(\chi\left(T_{i}\right)\right)^{n}\left(1-\chi\left(T_{i}\right)\right)^{M-n}
$$

where

$$
\chi\left(T_{i}\right)=\frac{\exp \left(-T_{i}\right)}{1+\exp \left(-T_{i}\right)}=\frac{1}{1+\exp \left(T_{i}\right)}
$$

We can then derive the mean of this distribution as follows:

$$
\begin{aligned}
\mathbb{E}\left(u_{i} \mid u_{j}, j \in N_{i}\right) & =M \chi\left(T_{i}\right) \\
& =\frac{M}{1+\exp \left(\beta_{1 i}+\beta_{2 i} \sum_{j \in N_{i}} u_{j}\right)}
\end{aligned}
$$

Then using Mean Field approximation [20] we can derive the average traffic intensity of node $i, \mathbb{E}\left(u_{i}\right)$ :

$$
\mathbb{E}\left(u_{i}\right) \approx \frac{M}{1+\exp \left(\beta_{1 i}+\beta_{2 i} \sum_{j \in N_{i}} \mathbb{E}\left(u_{j}\right)\right)}
$$

In this paper, we aim to use the probabilistic relaying of traffic at each node presented in Section IV as an alternative to the shortest path routing protocol, without the burden of routing table overheads. It is well known that with shortest path routing protocol, traffic transmitted by a node decreases with increasing distance from the center of the network [21]. This is due to the fact that nodes close to the center of the network are more likely to be on the shortest path between any two randomly chosen pair of nodes in the network. Hence such nodes have to retransmit more traffic coming from their neighbors. To guarantee these conditions, $\beta_{2 r}$ need to have two properties: 
Property 1: $\beta_{2 r} \leq 0$.

In equation (9), one can see that if the parameter $\beta_{2 i}$ is negative, the average traffic intensity $\mathbb{E}\left(u_{i}\right)$ of a node will increase with the traffic intensity average of its neighbors (i.e, we guarantee that the more traffic a node receives from its neighbors, the more traffic it will retransmit).

Property 2: The absolute value of $\beta_{2 i}$ increases as node $i$ gets closer to the center of the network.

Again, from equation (9), this property guarantees that the amount of traffic nodes relay from their neighbors increases as they get closer to the center of the network, as it is the case for the shortest path algorithm.

The objective of the next section is to estimate the $\beta_{1 i}$ and $\beta_{2 i}$ values so that the probabilistic model described above accurately mimics the quantitative behavior of the shortest path routing protocol in terms of traffic intensity.

\section{B. Parameter Estimation}

In this section we describe the simulation studies used to estimate the parameters $\beta_{1 i}$ and $\beta_{2 i}$. For simplicity a network of $N^{2}$ nodes located on a perfect grid with as shown in Figure 2 was used. Distance between nodes is set to $400 \mathrm{~m}$. These nodes use 802.11a MAC/PHY layer and Dynamic Source Routing (DSR) [4] ad hoc routing protocol. Transmission rate of all nodes are set to $1 \mathrm{Mbps}$ and transmitter power and the received power threshold of all nodes are set such that they can receive from nodes $400 \mathrm{~m}$ away but cannot receive from nodes that are diagonally across. Each node randomly picks a destination node and transmits a fixed length data packet to that node. The interarrival time for the packet generation process at each node is $100 \mathrm{~ms}$ and the packet generation rate at each node is set to $\lambda$.

With respect to the number of neighbours it has and its position in the network topology, all nodes in the network can be grouped into rings as shown in Figure 2. This simplifies the calculations as we only require one set of calculations for all nodes in a given ring.

As all nodes have the same packet generation rate the parameters $\beta_{1 i}$ will be a constant $\beta_{1}$ across the network. We have seen in the previous section that $\beta_{2 i}$ will have an impact on the amount of traffic that a node will relay from its neighbours, and we have also noted that this amount of traffic depends on the distance between this node and the center of the network. Therefore, all nodes in a given ring around the center of the network have the same $\beta_{2}$. Hence we can simplify $\beta_{2 i}$ to $\beta_{2 r} r=[1,2, \ldots]$.

Observing the above mentioned symmetry of the network, we can rewrite equation (9) as follows:

$$
\mathbb{E}\left(u_{r}\right)=\frac{M}{1+\exp \left(\beta_{1}+\beta_{2 r} \sum_{j \in N_{i}} \mathbb{E}\left(u_{j}\right)\right)}
$$

where $r$ is the ring number.

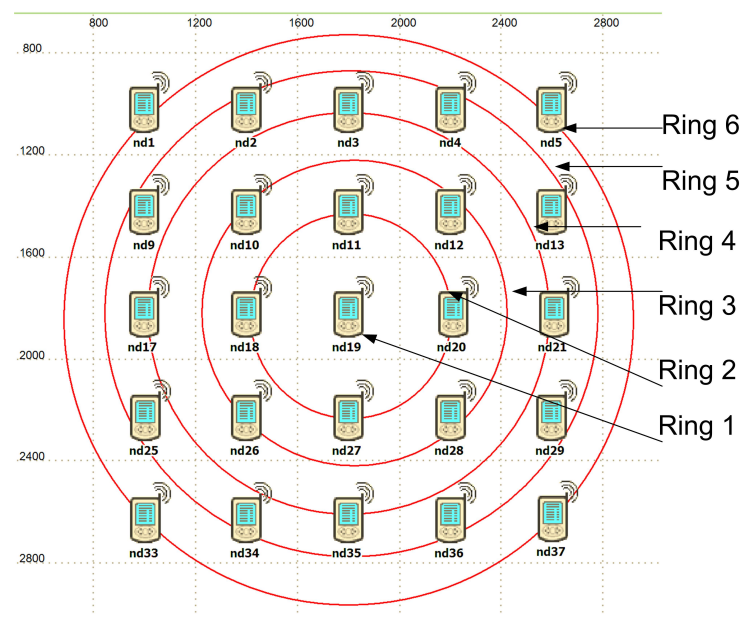

Fig. 2. Network topology

Let us assume that $\mathbb{E}\left(u_{j}\right) \approx \mathbb{E}\left(u_{r}\right)$ for $j \in N_{i}$, and recall that in our network model, each node has 4 neighbors. Then, substituting $X_{r}=M / \mathbb{E}\left(u_{r}\right)$ into equation (10) and reordering:

$$
\beta_{2 r}=\frac{X_{r}\left(\log \left(X_{r}-1\right)-\beta_{1}\right)}{4 M}
$$

1) Large Networks: When the network is large enough, for shortest path routing, the average traffic intensity on each ring $r$ can be estimated as follows according to [21]:

$$
\mathbb{E}\left(u_{r}\right)=\lambda+\lambda_{1}\left(1-\frac{l_{r}^{2}}{l_{R}^{2}}\right)^{2}
$$

where $l_{k}$ is the radius of the $k^{t h}$ ring in the network, $R$ is the largest ring which contains boundary nodes, $\lambda$ is the traffic generation rate of each node, and $\lambda_{1}$ is a parameter that depends on the network density.

Note that nodes at the boundary of the network $(r=R)$ retransmit a very small amount of traffic from their neighbors as they are not likely to be on the shortest path between any two nodes. Therefore, we set $\beta_{2 r} \approx 0$ for $r=R$. From equation (10) and using the fact that $X_{r}=M / \lambda$, for $r=R$ we have:

$$
\beta_{1}=\log \left(\frac{M}{\lambda}-1\right)
$$

For $r>0, \beta_{2 r}$ can then be easily calculated using equation (11) with $X_{r}$ calculated as:

$$
X_{r}=\frac{M}{\lambda+\lambda_{1}\left(1-\frac{l_{r}^{2}}{l_{R}^{2}}\right)^{2}}
$$

Note that in the above equations, in order to calculate the parameters $\beta_{1}$ and $\beta_{2 r}$, we only need to know the size of the network and the traffic generation rate of each node. 
2) Small Networks: As mentioned above, equation (12) is only applicable to large networks. For smaller networks, such as the one in Figure 2, we use shortest path simulation results to estimate these parameters, i.e, we use empirical results for $E\left[u_{r}\right]$ for each ring. An important issue is to ensure that these parameters are such that Property 1 and Property 2 explained in Section III-A hold, i.e, that $\beta_{2 r}$ is strictly negative for all rings and increases with $r$. Note that $X_{r}$ increases with $r$. We will therefore choose $\beta_{1}$ such that the function;

$$
\beta_{2 r}=f\left(X_{r}\right)=X_{r}\left(\log \left(X_{r}-1\right)-\beta_{1}\right) /(4 M)
$$

is negative and increases with $X_{r}$. It is easy to see that $\beta_{2 r}$ is negative if and only if:

$$
\beta_{1}>\log \left(X_{r}-1\right)
$$

Now, taking the derivative the $f\left(X_{r}\right)$ with respect to $X_{r}$ and imposing that this derivative be strictly positive for all $X_{r}$, leads to the following condition on $\beta_{1}$ ensuring that $\beta_{2 r}$ is an increasing function:

$$
\beta_{1}<\frac{X_{r}}{X_{r}-1}+\log \left(X_{r}-1\right)
$$

We therefore need to find a suitable $\beta_{1}$ such that the following inequality is verified for every ring $r$ :

$$
\log \left(X_{r}-1\right)<\beta_{1}<\frac{X_{r}}{X_{r}-1}+\log \left(X_{r}-1\right)
$$

To estimate $\beta_{1}$, the above described network $(N=4,5)$ was simulated for 3000s with different $\lambda$ values and 10 simulations runs were conducted for each $\lambda$ value. Then from the simulation results the average traffic intensity (total sending rate) at a node in each of the rings, $\mathbb{E}\left(u_{r}\right)$, was estimated for each $\lambda$ value. For each of these simulation scenarios we observed that;

$$
\min \left(\frac{X_{r}}{X_{r}-1}+\log \left(X_{r}-1\right)\right)>\log \left(X_{r}-1\right) \forall r
$$

Hence, we set $\beta_{1}=\min \left(\frac{X_{r}}{X_{r}-1}+\log \left(X_{r}-1\right)\right)$. Then $\beta_{2}$ was estimated from equation (10). Having estimated the necessary parameters, we now propose our probabilistic relaying scheme which will provide the same traffic pattern as the shortest path routing.

\section{Probabilistic Relaying}

The main objective of the probabilistic relaying process is to remove the complex routing algorithms at the routing layer and introduce a simple retransmission mechanism which results in similar performance. As described earlier, our framework requires that the traffic intensity at nodes should be integer values between 0 and $M$. To achieve this we divide the system time into $100 \mathrm{~ms}$ blocks and impose a condition that traffic transmitted at the routing layer during $100 \mathrm{~ms}$ interval should be integer values $0,1, \ldots, 200 \mathrm{kbps}$. This implies that $M=200$.

Assuming no packets are lost during the transmission, the traffic received at a node should be equal to the sum of traffic transmitted by all neighbouring nodes. Hence we can replace $\sum_{j \in N_{i}} \mathbb{E}\left(u_{j}\right)$ in equation (10) with the traffic received at node $i, R_{i}$. Once $R_{i}$ is known the conditional probability distribution for traffic transmitted, given the traffic transmitted by its neighbours is given by the binomial cumulative distribution function on $0 \ldots M$ with parameters $M$ and $\chi\left(T_{i}\right)$. Note that these conditional binomial distributions can be generated for all rings in the networks for all possible received traffic values and stored in look up tables. Given that the maximum number of neighbours a node can have in this topology is 4 , the maximum traffic a node can receive is $800 \mathrm{kbps}$. Hence the size of the distribution function at each node is a $800 \times 200$ element matrix. Let us call this matrix $T R_{r}$.

We add an extra field to the routing header, called hop addresses, which carries the address of all nodes that the packet has traversed up to that point. Once a packet has reached the routing layer from the application layer, the routing layer will add its address to the first element of the hop address field and then that packet will be stored at the routing layer. All packets received by the MAC layer will be sent to the routing layer where the algorithm given in Figure 3 is used to decide whether the packet should rebroadcasted unless the packet has reached its final destination. The first check in this algorithm avoids transmission loops while the second check removes old packets from the network. MAX_HOPS is the maximum number of hops a packet is allowed to travel in the network. This should be selected such that it is large enough for packets from any nodes to reach any other node in the network while small enough to prevent unnecessary retransmission of packets. This acts as a time to live field in IP packets.

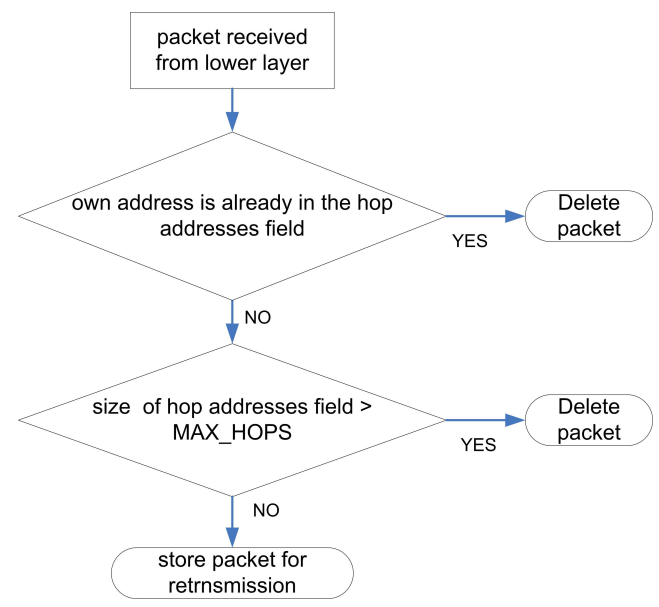

Fig. 3. Processing packets received from the MAC layer

Nodes transmit packets from the routing layer every 100 ms. This value should be selected to satisfy the delay requirements of the network. In this work we have not imposed any delay requirement for the network. Nodes calculate the amount of traffic received, $R_{i}$, at the IP layer during the $100 \mathrm{~ms}$ between 2 transmission opportunities. Once $R_{i}$ is known, a node will generate a random number between 0 and 1 from a uniform distribution. Then the node will estimate the amount of traffic it should transmit from the routing layer as the traffic intensity which corresponds to cumulative probability greater than the generated random number in the $R_{i}^{\text {th }}$ column of the $T R_{r}$ matrix. Estimating the traffic intensity at each node in 
this manner guarantees over time that the traffic intensity of each node, conditional on the traffic intensity of neighbours is binomial with appropriate parameters. At the MAC layer, packets are transmitted using 802.11 MAC protocol. In this paper, the effect of the MAC layer on the routing behaviour is minimised by keeping the routing layer transmission rates well below what is supported by the MAC and physical layers.

Once the amount of data that should be sent to the MAC layer is is estimated, the routing layer forwards the appropriate amount of packets to the MAC layer. Any left over packets at the routing layer, after forwarding the appropriate amount to the MAC layer, are discarded. Just before forwarding to the MAC layer, packets are reordered at the routing layer. At the head of the queue will be packets generated by the node, ordered according to the packet generation time. All relay packets are ordered according to the relay node's proximity to the shortest path between the packet's source and destination. This requires all the nodes to know the location of other nodes. In a sensor network application with no mobility or ad hoc networks with low mobility this could be achieved by periodic dissimilation of node locations throughout the network. In this work we use two extra fields in the routing header to carry the source and destination location of a packet.

\section{Simulations And Results}

Probabilistic relaying process as described in Section IV was implemented at the routing layer of the sensor nodes. Similar to the shortest path simulation all nodes were organised on a symmetrical grid pattern as shown in Figure 2. This network was then simulated for 6000s under the traffic generation profiles identical to that used in shortest path simulations. All the results obtained below were averaged over 10 simulation runs.

Figures 4 and 5 respectively show the $\beta_{1}$ and $\beta_{2}$ values estimated from shortest path and probabilistic relaying simulation results for a 16 node network. In these figures "SP" refers to shortest path and "PR" refer to probabilistic relaying. It can be observed in both figures that the all $\beta$ values match well for the two cases. This confirms that the traffic intensity patterns in the probabilistic relaying and shortest path simulations closely resembles each other. Hence we can conclude that the proposed method is capable of closely recreating the overall traffic patterns expected if shortest path was used in the network.

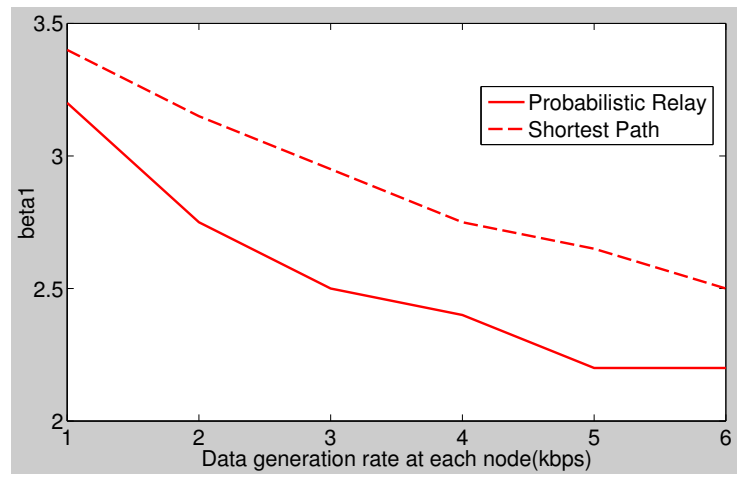

Fig. 4. $\quad \beta_{1}$ estimated from shortest path and probabilistic relaying simulations results for 16 node network

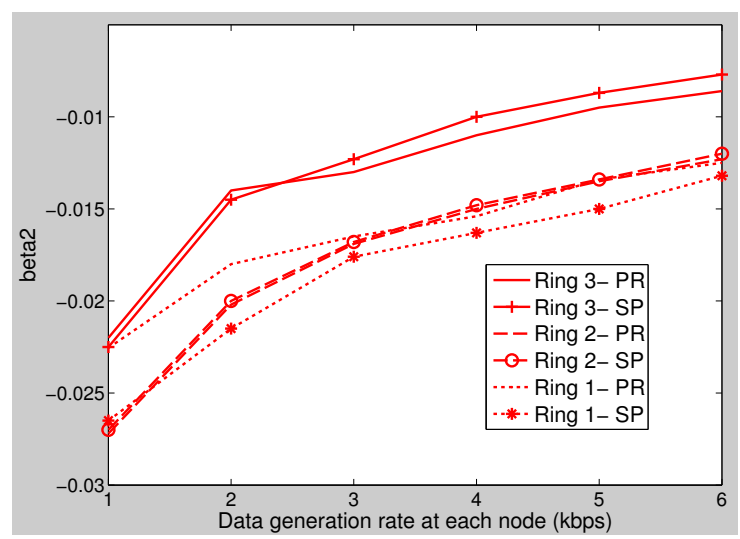

Fig. 5. $\beta_{2}$ estimated from shortest path and probabilistic relaying simulations results for 16 node network

Figure 6 compares the throughput performance of the probabilistic relaying approach to that of shortest path. It can be observed that the throughput from the proposed scheme is less than $5 \%$ below the shortest path throughput. A similar trend can be observed for results for a 25 node network shown in Figure 7. From these results we can conclude that the proposed probabilistic relaying approach is able to closely emulate the performance of shortest path routing without the complex routing procedures or the excessive control message overhead commonly associated with routing.

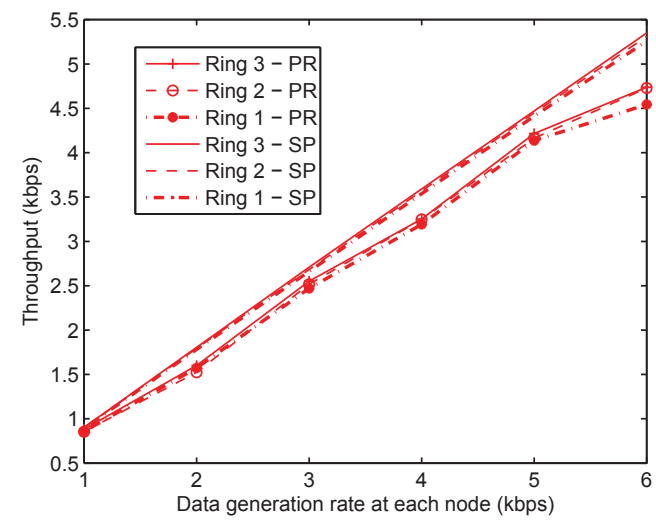

Fig. 6. Average throughput for 16 node network

\section{CONCLUSION}

Lack of fundamental understanding of the complex interactions between neighbouring nodes in ad hoc and sensor networks is a significant impediment for practical deployment of such networks. In this paper we propose a framework based on MRFs for analysis and design of multihop networks. MRFs are used to analyse large distributed systems with strong spatial dependencies as demonstrates in image processing applications.

In this paper we used Markov Random Fields to model the traffic intensity of nodes in a multihop network using shortest path routing. It was demonstrated that the conditional distribution of traffic at each node can be characterised using 


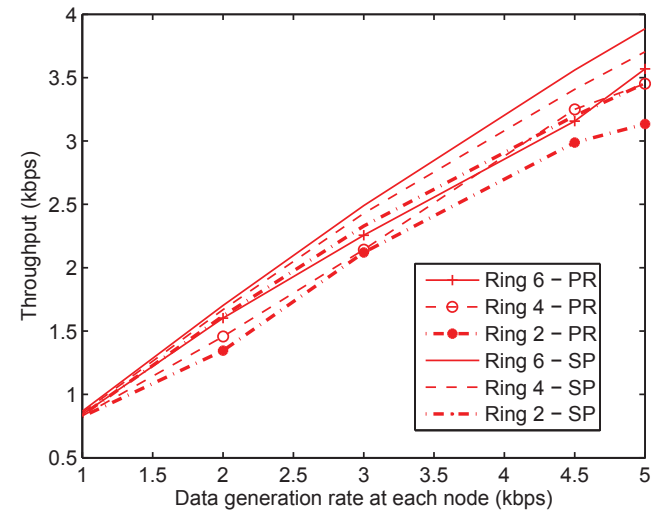

Fig. 7. Average throughput for 25 node network

a binomial distribution of two parameters, one describing the traffic generated at each node and the other describing the relationship between traffic intensities at neighbouring nodes. Later we proposed a probabilistic relaying mechanism to recreate the traffic distribution similar to that for shortest path routing. The objective is to recreate the shortest path routing behaviour without using complex multihop routing mechanisms and associated overhead. Using an OPNET based simulation study we have shown that the traffic intensity pattern for the proposed mechanism is similar to that for shortest path routing. Furthermore, it was shown that throughput performance of the proposed mechanism is within $95 \%$ of what is achieved in shortest path for all scenarios.

One drawback of this mechanism is the amount of unnecessary packets created in the network during the relaying process. Currently we are investigating how a mechanism like network coding can be used to reduce these packets. In [22] we prosed a power control framework based on MRF to guarantee connectivity of nodes in a arbitrary sensor network. We intend to combine these two models, power control model and traffic intensity model, to extend the current traffic intensity model to arbitrary networks.

\section{REFERENCES}

[1] S. Kumar, V. S. Raghavan, and J. Deng, "Medium Access Control Protocols for Ad hoc Wireless Networks: A Survey," Ad Hoc Networks, vol. 4, no. 3, pp. 326 - 358, 2006.

[2] Y.-B. Ko, V. Shankarkumar, and N. Vaidya, "Medium Access Control Protocols Using Directional Antennas in Ad hoc Networks," in INFOCOM 2000. Nineteenth Annual Joint Conference of the IEEE Computer and Communications Societies. Proceedings. IEEE, vol. 1, 2000, pp. 13-21 vol.1.

[3] E. Royer and C. Toh, "A Review of Current Routing Protocols for AdHoc Mobile Wireless Networks," IEEE Personal Communications, pp. 1070-1099, April 1999.

[4] D. Johnson and D. Maltz, "Dynamic Source Routing in Ad Hoc Wireless Networks," in Mobile Computing, T. Imielinkski and H. Korth, Eds. Kluwer Academic Publishers, 1996.

[5] G. Holland and N. Vaidya, "Analysis of TCP performance over Mobile Ad hoc Networks," Wirel. Netw., vol. 8, no. 2/3, pp. 275-288, 2002.

[6] K. Sundaresan, V. Anantharaman, H.-Y. Hsieh, and A. Sivakumar, "ATP: A Reliable Transport Protocol for Ad hoc Networks," Mobile Computing, IEEE Transactions on, vol. 4, no. 6, pp. 588-603, Nov.Dec. 2005.
[7] T. He, C. Huang, B. M. Blum, J. A. Stankovic, and T. Abdelzaher, "Range-free Localization Shemes for Large Scale Sensor Networks," in MobiCom '03: Proceedings of the 9th annual international conference on Mobile computing and networking. New York, NY, USA: ACM, 2003, pp. 81-95.

[8] M. Conti, G. Maselli, G. Turi, and S. Giordano, "Cross-Layering in Mobile Ad hoc Network Design," Computer, vol. 37, no. 2, pp. 48-51, Feb 2004.

[9] J. Broch, D. A. Maltz, D. B. Johnson, Y.-C. Hu, and J. Jetcheva, "A Performance Comparison of Multihop Wireless Ad hoc Network Routing Protocols," in MobiCom '98: Proceedings of the 4th annual ACM/IEEE international conference on Mobile computing and networking. New York, NY, USA: ACM, 1998, pp. 85-97.

[10] P. Gupta and P. Kumar, "The Capacity of Wireless Networks," Information Theory, IEEE Transactions on, vol. 46, no. 2, pp. 388-404, Mar 2000.

[11] A. Jayasuriya, S. Perreau, A. Dadej, and S. Gordon, "Hidden vs. exposed terminal problem in ad hoc networks," in Australian Telecommunication Networks and Applications Conference (ATNAC 2004), 2004.

[12] Y. Liaw, A. Dadej, and A. Jayasuriya, "Throughput Performance of Multiple Independent Paths in Wireless Multihop Networks," in Communications, 2004 IEEE International Conference on, vol. 7, June 2004, pp. 4157-4161 Vol.7.

[13] A. Muqattash and M. Krunz, "CDMA-based MAC Protocol for Wireless Ad hoc Networks," in MobiHoc '03: Proceedings of the 4th ACM international symposium on Mobile ad hoc networking \& computing. New York, NY, USA: ACM, 2003, pp. 153-164.

[14] X. Descombes, M. Sigelle, and F. Preteux, "Estimating Gaussian Markov Random Fields Parameters in a Non-stationary Framework: Application to Remote Sensing Imaging," IEEE Transactions on Image Processing, vol. 8, no. 4, pp. 490-503, 199.

[15] R. Chellappa and S. Chatterjee, "Classification of Textures Using Gaussian Markov Random Fields," Acoustics, Speech and Signal Processing, IEEE Transactions on, vol. 33, no. 4, pp. 959-963, Aug 1985.

[16] R. Chellappa and A. Jain, Markov Random Fields: Theory and Application, R. Chellappa, Ed. Boston: Academic Press, 1993.

[17] A. Dogandzic and B. Zhang, "Distributed Estimation and Detection for Sensor Networks Using Hidden Markov Random Field Models," Signal Processing, IEEE Transactions on, vol. 54, no. 8, pp. 3200-3215, Aug. 2006.

[18] L. Doyle, A. Kokaram, S. Doyle, and T. Forde, "Ad Hoc Networking, Random Markov Fields and Decision Making," IEEE Signal Processing Magazine, pp. 63-73, Septmember 2006.

[19] S. Jeong and C. Ji, "Randomized Distributed Configuration Management of Wireless Networks: Multi-Layer Markov Random Fields and Near Optimality," submitted the IEEE Trans. on Networking, 2008. [Online]. Available: http://arxiv.org/abs/0809.1916

[20] J. Zhang, "The Mean Field Theory in EM Procedures for Markov Random Fields," Signal Processing, IEEE Transactions on, vol. 40, no. 10, pp. 2570-2583, Oct 1992.

[21] P. Pham and S. Perreau, "Analysis of Reactive Shortest Single-path Routing Mechanism Versus Multipath Routing Protocol with Load Balance Policy," in Workshop on the Internet, Telecommunications and Signal Processing, 2002.

[22] S. Perreau, M. Sigelle, P. Da Silva, and A. Jayasuriya, "Power Control and Resource Allocation in Sensor Networks Using Random Markov Field Theory," in IEEE Sencon, Rome, Italy, 2009. 